\title{
Model for the Scaling of Stresses and Fluctuations in Flows near Jamming
}

\author{
Brian P. Tighe, ${ }^{1}$ Erik Woldhuis, ${ }^{1}$ Joris J.C. Remmers, ${ }^{1,2}$ Wim van Saarloos, ${ }^{1}$ and Martin van Hecke ${ }^{3}$ \\ ${ }^{1}$ Instituut-Lorentz, Universiteit Leiden, Postbus 9506, 2300 RA Leiden, The Netherlands \\ ${ }_{2}^{2}$ Eindhoven University of Technology, Postbus 513, 5600 MB, Eindhoven, The Netherlands \\ 3 Kamerlingh Onnes Lab, Universiteit Leiden, Postbus 9504, 2300 RA Leiden, The Netherlands
}

(Dated: October 24, 2018)

\begin{abstract}
We probe flows of soft, viscous spheres near the jamming point, which acts as a critical point for static soft spheres. Starting from energy considerations, we find nontrivial scaling of velocity fluctuations with strain rate. Combining this scaling with insights from jamming, we arrive at an analytical model that predicts four distinct regimes of flow, each characterized by rational-valued scaling exponents. Both the number of regimes and values of the exponents depart from prior results. We validate predictions of the model with simulations.
\end{abstract}

PACS numbers: 47.57.Bc, 83.50.Rp, 83.80.Iz

The past few years have seen enormous progress towards understanding the static, "jammed" state that occurs when soft athermal particles are packed sufficiently densely that they attain a finite rigidity $[1-3$. Such systems may flow when shear stresses are applied, and in seminal work, Olsson and Teitel addressed the relation between strain rate, shear stress and packing fraction in a simplified numerical model for the flow of soft viscous spheres [4. When rescaled appropriately, the data for strain rate $\dot{\gamma}$, shear stress $\sigma$ and packing fraction $\phi$ were found to collapse to two curves, reminiscent of second order-like scaling functions, and a large length scale was found to emerge near jamming. Since then, qualitatively similar results have been obtained in simulations of a number of flowing systems [5] 9], but there is little agreement on the actual value of scaling exponents, nor on the relation to jamming in static systems.

Here we describe an analytical model that connects the scaling of static systems to the scaling of both the velocity fluctuations and the shear stress of flowing systems near jamming. The model is built around a "viscoplastic" effective strain $\gamma_{\text {eff }}=\gamma_{\mathrm{y}}+\gamma_{\mathrm{dyn}}$, where $\gamma_{\mathrm{dyn}}$ is a dynamic contribution set by the strain rate, and $\gamma_{\mathrm{y}}$ stems from the (dynamical) yield stress and is controlled by the distance to jamming. We show that steady state power balance dictates nontrivial scaling of $\gamma_{\text {dyn }}$ with strain rate, and propose a nonlinear stress-strain relation that leads to a closed set of equations predicting a rich scaling scenario for flows near jamming. We verify central ingredients of the model and our predictions for the rheology numerically in Durian's bubble model for foams [11. Our simple model captures and predicts the rheology and fluctuations starting from the microscopic interactions; it also indicates the need for, and provides, new ways to present and analyze rheological data near jamming.

Numerical Model - The two-dimensional Durian bubble model stipulates overdamped dynamics in which the sums of elastic and dissipative forces on each bubble, represented by a disk, balance at all times [11. Forces are pairwise and occur only between contacting bubbles.

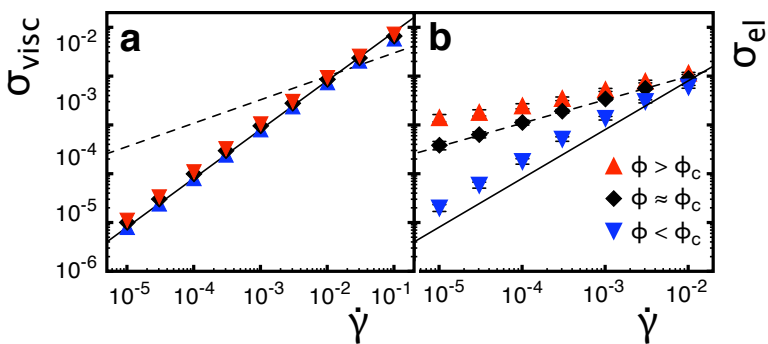

FIG. 1: Viscous stress (a) and elastic stress (b) for packing fractions $\phi=0.80,0.8424$, and 0.87 . The dashed line $\sim \dot{\gamma}^{0.48}$. $\sigma_{\text {visc }}$ scales linearly with $\dot{\gamma}$ (solid line) and lies below the elastic stresses for $\dot{\gamma}<\mathcal{O}\left(10^{-2}\right)$.

Elastic interaction forces are proportional to the disk overlap, $f_{i j}^{\mathrm{el}}=k\left(R_{i}+R_{j}-r_{i j}\right)^{\alpha_{\mathrm{el}}}$, where $\vec{r}_{i j}:=\vec{r}_{j}-\vec{r}_{i}$ points from one bubble center to another and $R_{i}$ labels the radius of disk $i$. In the full model that we focus on here, viscous forces oppose the bubbles' relative velocity $\Delta \vec{v}_{i j}:=\vec{v}_{j}-\vec{v}_{i}$ with magnitude $f_{i j}^{\text {visc }}=b\left|\Delta v_{i j}\right|^{\alpha_{\text {visc }}}$ [12. In simulations we set both exponents $\alpha_{\mathrm{el}}$ and $\alpha_{\mathrm{visc}}$ to unity. The strain rate $\dot{\gamma}$ is imposed via Lees-Edwards boundary conditions. The unit cell contains a 50:50 bidisperse mixture of $N=1020$ to 1210 bubbles with size ratio 1.4:1. Stresses are averaged over a run (total time $20 / \dot{\gamma}$ ) after discarding the transient.

Elastic and Viscous Stress - Because forces balance on each bubble, the shear stress can be computed according to $\sigma_{x y}:=\sigma_{\text {tot }}=\frac{1}{2 V} \sum_{\langle i j\rangle} r_{i j, x}\left(f_{i j, y}^{\text {el }}+f_{i j, y}^{\text {visc }}\right)$, where $V$ is the area of the unit cell and the sum runs over contacting pairs. It is convenient to distinguish contributions of elastic and viscous forces to the total stress $\sigma_{\text {tot }}:=\sigma_{\text {el }}+\sigma_{\text {visc }}$. Fig. 11 depicts $\sigma_{\text {el }}$ and $\sigma_{\text {visc }}$ as functions of strain rate for three packing fractions. We find that the viscous stress $\sigma_{\text {visc }}$ depends only weakly on $\phi$ and scales linearly with $\dot{\gamma}$, and dominates the total stress for strain rates $\dot{\gamma} \gtrsim \mathcal{O}\left(10^{-2}\right)$. We denote this regime as Viscous $(\mathrm{V})$, and since $\sigma_{\text {visc }} \sim \dot{\gamma}, \sigma_{\text {tot }} \sim \dot{\gamma}$ here. Our analytical model, developed below, treats the case where $\sigma_{\text {el }}$ dominates the stress, and in all that follows, $\dot{\gamma} \leq 10^{-2}$, $\sigma_{\mathrm{visc}} \ll \sigma_{\mathrm{el}}, \sigma_{\mathrm{tot}} \approx \sigma_{\mathrm{el}}$, and we no longer distinguish 


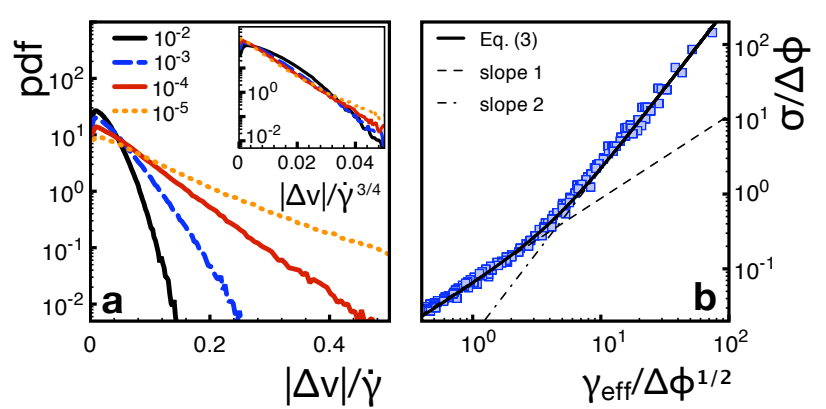

FIG. 2: (a) The probability distribution function (pdf) of relative bubble velocities $|\Delta v|$, for $\phi \approx \phi_{c}$ and strain rates $\dot{\gamma}$ spanning three decades (see legend), does not collapse when rescaled by $\dot{\gamma}$. In contrast, the pdf of $|\Delta v| / \dot{\gamma}^{3 / 4}$ yields a reasonable collapse (inset). Note that Eq. (1) predicts the pdf's second moment, not its shape. (b) Collapsed stress-effective strain relation for $\gamma_{\text {eff }} \propto \Delta \phi+0.7(\dot{\gamma} / \sigma)^{1 / 2}$ and the same data as in Fig. 4 The solid curve is $y=0.085 x \sqrt{1+0.05 x^{2}}$.

between $\sigma_{\text {tot }}$ and $\sigma_{\text {el }}$, referring to both as as $\sigma$.

Main Phenomenology - As Fig. 1 $1 \mathrm{~b}$ illustrates, the rheology is nontrivial and departs from simple linear scalings. For $\phi$ above $\phi_{c}=0.8423 \pm 0.001$ [13], the stress flattens as $\dot{\gamma}$ is lowered, while for $\phi \approx \phi_{c}$ the stress is welldescribed by a power law $\sigma \sim \dot{\gamma}^{1 / 2}$ — this will emerge below as the Critical scaling regime of the rheology. For $\phi$ below $\phi_{c}$, the stress shows increasing downward curvature as strain rate is decreased; our model does not treat this case, and we do not consider it further.

Analytical Model - To relate stress and strain rate, we will (i) find expressions for the dynamic and yield strains $\gamma_{\mathrm{y}}$ and $\gamma_{\text {dyn }}$ that constitute the effective strain and connect them to the stress via power balance; (ii) express the stress as a function of $\gamma_{\text {eff }}$.

(i) The effective strain $\gamma_{\text {dyn }}$ can be thought of as the typical strain undergone between plastic rearrangements of the contact network. Because bubbles move with relative velocity $|\Delta v|$, the contact network rearranges on a time scale $t_{\text {dyn }} \sim \bar{d} /|\Delta v|$, where $\bar{d}$ is the average bubble diameter. The typical strain incurred on this time scale is $\gamma_{\text {dyn }}:=\dot{\gamma} t_{\text {dyn }} \sim \dot{\gamma} /|\Delta v|$.

To obtain an estimate for $|\Delta v|$, we turn our attention to the relation between the scaling of stress and velocity fluctuations. As noted in Ref. [14], mechanical energy is supplied to the system at a rate $\sim \sigma_{\text {tot }} \dot{\gamma}$. Energy dissipation takes place by bubbles moving past each other - hence the dissipation rate scales as $f^{\text {visc }}|\Delta v| \sim|\Delta v|^{2}$ [15. Balancing the two yields

$$
\sigma_{\text {tot }} \dot{\gamma} \sim|\Delta v|^{2}
$$

Eq. (1) is the first of three relations comprising our model: given the stress, it provides the scaling of $|\Delta v|$.

It will emerge from our model that the nontrivial scaling of velocity fluctuations underlies the rich rheology. Fig. 2 a shows probability distributions of $|\Delta v| / \dot{\gamma}$ for $\phi \approx \phi_{c}$ and $\dot{\gamma} \leq 10^{-2}$. Since here $\sigma \sim \dot{\gamma}^{1 / 2},|\Delta v|$ does not scale as $\dot{\gamma}$. In fact, Eq. (1) predicts that in this case

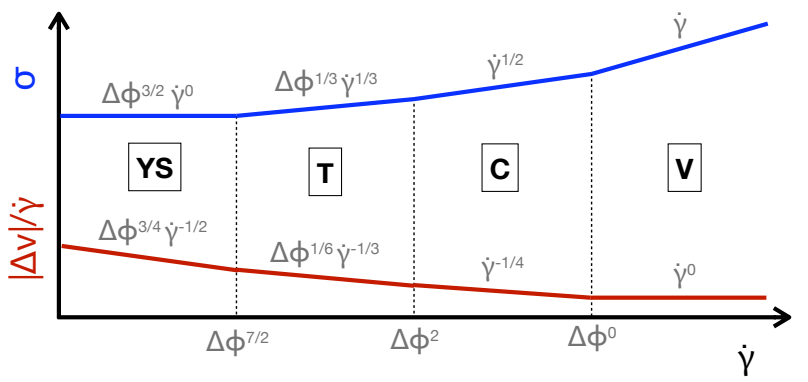

FIG. 3: Schematic depiction of predictions for scaling of stress $\sigma$ and fluctuations in the relative bubble motion $|\Delta v| / \dot{\gamma}$ with strain rate $\dot{\gamma}$ and distance to the critical packing fraction $\Delta \phi=\phi-\phi_{c}$. There are four distinct flow regimes: Yield Stress (YS), Transition (T), Critical (C) and Viscous (V).

$|\Delta v| \sim \dot{\gamma}^{3 / 4}$. This gives a good collapse of the data (inset Fig. 2 a). Note that in the Viscous regime where $\sigma \sim \dot{\gamma}$, one finds that $|\Delta v|^{2} \sim \dot{\gamma}^{2}$, so that the typical relative velocity $|\Delta v|$ scales trivially with $\dot{\gamma}$ (not shown).

For $\phi>\phi_{c}$ one anticipates a threshold (yield) stress even for vanishingly low strain rate, which in our picture translates into an additional contribution to the effective strain; this is $\gamma_{\mathrm{y}}$. A reasonable expectation for the scaling of $\gamma_{\mathrm{y}}$ is the strain scale required to prepare a packing at $\phi=\phi_{c}+\Delta \phi$ by compressing a system from the critical packing fraction: $\gamma_{\mathrm{y}} \sim \Delta \phi / \phi \sim \Delta \phi$. Collecting terms, the effective strain reads:

$$
\gamma_{\mathrm{eff}}=A_{1} \Delta \phi+A_{2} \bar{d} \dot{\gamma} /|\Delta v|
$$

(ii) We now construct a stress-strain relation $\sigma=$ $g\left(\Delta \phi, \gamma_{\text {eff }}\right) \gamma_{\text {eff }}$ and make the ansatz that the shear modulus $g$ displays single parameter scaling: $g\left(\Delta \phi, \gamma_{\text {eff }}\right)=$ $\Delta \phi^{p} \tilde{g}\left(\gamma_{\mathrm{eff}} / \Delta \phi^{q}\right)$. We will determine a form of $\tilde{g}$ based on known results for static systems. Above $\phi_{c}$, static systems display a regime of linear response $\sigma=G \gamma$, where the static shear modulus $G=G_{0} \sqrt{\Delta \phi}[16]$. Hence $p=1 / 2$ and $\tilde{g}(x) \rightarrow G_{0}$ for $x \rightarrow 0$. Precisely at $\phi_{c}, G$ vanishes, no analytic expansion of the stress-strain relation is possible, and critical static spring networks display the quadratic form $\sigma=\kappa\left|\gamma_{\text {eff }}\right| \gamma_{\text {eff }}$ [17]. It follows that $q=1 / 2$ and $\tilde{g}(x) \rightarrow \kappa|x|$ for $x \rightarrow \infty$. Therefore the stress-strain relation can be rewritten as

$$
\frac{\sigma}{\Delta \phi}=\tilde{g}\left(\frac{\gamma_{\mathrm{eff}}}{\sqrt{\Delta \phi}}\right) \frac{\gamma_{\mathrm{eff}}}{\sqrt{\Delta \phi}} .
$$

In the analysis to follow, only $p, q$, and the asymptotic scaling of $\tilde{g}(x)$ are essential. In Fig. 2b we show that a scatter plot of $\sigma / \Delta \phi$ as function of $\gamma_{\text {eff }} / \sqrt{\Delta \phi}$ shows excellent data collapse with the correct asymptotic behavior - here $\gamma_{\text {eff }} \propto \Delta \phi+\left(A_{2} \bar{d} / A_{1}\right)(\dot{\gamma} / \sigma)^{1 / 2}$ and $A_{2} / A_{1}$ has been adjusted to obtain collapse. We also note that the simplest choice for $\tilde{g}(x)$ that obeys reflection symmetry, remains analytic above $\phi_{c}$ and obeys all necessary scalings is $\tilde{g}(x)=G_{0} \sqrt{1+\left(\kappa x / G_{0}\right)^{2}}$, which fits the data remarkably well (Fig. $2 \mathrm{~b})$. 


\begin{tabular}{|c|c|c|c|c|}
\hline & Yield Stress & Transition & Critical & Viscous \\
\hline Stress & & $\sigma \approx \sigma_{\mathrm{el}}$ & & $\sigma \approx \sigma_{\text {visc }}$ \\
\hline Model & $\left\{\begin{aligned} \sigma \dot{\gamma} & \sim|\Delta v|^{2} \\
\gamma_{\mathrm{y}} & \sim \Delta \phi \\
\sigma & \sim G \gamma_{\mathrm{y}}\end{aligned}\right.$ & $\begin{cases}\sigma \dot{\gamma} & \sim|\Delta v|^{2} \\
\gamma_{\mathrm{dyn}} & \sim \dot{\gamma} /|\Delta v| \\
\sigma & \sim G \gamma_{\mathrm{dyn}}\end{cases}$ & $\left\{\begin{array}{l}\sigma \dot{\gamma} \sim|\Delta v|^{2} \\
\gamma_{\mathrm{dyn}} \sim \dot{\gamma} /|\Delta v| \\
\sigma\end{array}\right.$ & \\
\hline Result & 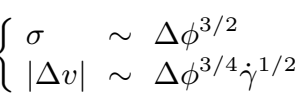 & $\begin{cases}\sigma & \sim \Delta \phi^{1 / 3} \dot{\gamma}^{1 / 3} \\
|\Delta v| & \sim \Delta \phi^{1 / 6} \dot{\gamma}^{2 / 3}\end{cases}$ & $\begin{cases}\sigma & \sim \dot{\gamma}^{1 / 2} \\
|\Delta v| & \sim \dot{\gamma}^{3 / 4}\end{cases}$ & $\begin{array}{ll}\sigma & \sim \dot{\gamma} \\
|\Delta v| & \sim \dot{\gamma}\end{array}$ \\
\hline Range & $\dot{\gamma}<\Delta \phi^{\frac{7}{2}}$ & $\Delta \phi^{\frac{7}{2}}<\dot{\gamma}<\Delta \phi^{2}$ & $\Delta \phi^{2}<\dot{\gamma}<0.01$ & $0.01<\dot{\gamma}$ \\
\hline
\end{tabular}

TABLE I: Analytical model and its solutions in the Yield Stress, Transition, Critical and Viscous regimes.

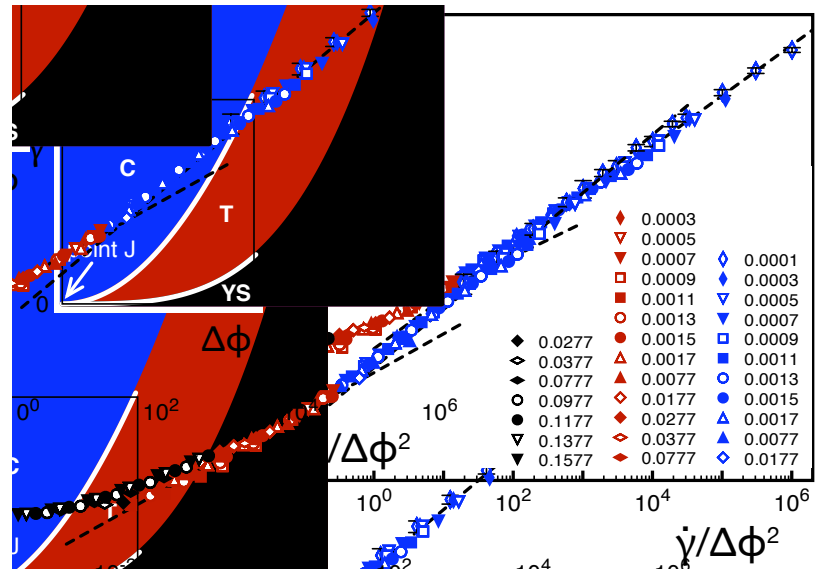

FIG. 4: Scaling collapse over four decades in strain rate $\dot{\gamma}$ and three decades in distance to the critical packing fraction $\Delta \phi$ (legend). Rescaled coordinates $\sigma / \Delta \phi$ and $\dot{\gamma} / \Delta \phi^{2}$ are appropriate for parameters spanning the Transition and Critical regimes (see Table I). Dashed lines are guides to the eye with slopes $1 / 3$ and $1 / 2$. (inset) Boundaries between the Yield Stress, Transition and Critical regimes in the $\Delta \phi-\dot{\gamma}$ plane.

Our model comprises Eqs. (1,3), which express $\sigma, \gamma_{\text {eff }}$, and $|\Delta v|$ in terms of $\dot{\gamma}$ and $\Delta \phi$. For scaling analysis the constants $A_{1}, A_{2}, G_{0}, \kappa$ and $\bar{d}$ can be set to unity.

Flow Regimes - The three equations for $\sigma, \gamma_{\text {eff }}$, and $|\Delta v|$ in terms of $\dot{\gamma}$ and $\Delta \phi$ lead to our scaling predictions. Eqs. (2) and (3) each have two scaling regimes, which are selected by varying $\dot{\gamma}$ and $\Delta \phi$. In combination, these contribute three scaling regimes to the rheology, i.e. the stress-strain rate relation. So where previous scaling ansätze presume two rheological regimes [4, 6, 8] - a yield stress plateau giving way to a power law in $\dot{\gamma}$ for higher strain rates — we find Yield Stress (YS), Transition (T), Critical (C) and Viscous (V) regimes, each persisting over a finite range of strain rates (Fig. 3). Table I collects the pertinent equations, solutions and parameter ranges for all scaling regimes.

Checking the Model - For data restricted to two regimes, it is possible to collapse the $\dot{\gamma}-\sigma$ flow curves to a master curve by rescaling with $\Delta \phi$. For the Transition and Critical regimes, the rheology is predicted to obey $\sigma \sim \Delta \phi^{\frac{1}{3}} \dot{\gamma}^{\frac{1}{3}}$ and $\sigma \sim \dot{\gamma}^{\frac{1}{2}}$, respectively. Hence for data in these two regimes, $\sigma / \Delta \phi$ vs $\dot{\gamma} / \Delta \phi^{2}$ can be collapsed to a master flow curve, characterized by a crossover from a $1 / 3$ to a $1 / 2$ power law scaling.
This strong test of the model is shown in Fig. 4, where data for three decades in $\Delta \phi$ and four decades in $\dot{\gamma}$ collapse to a single master curve. Data points satisfying $\dot{\gamma}<c_{1} \Delta \phi^{7 / 2}$ and $\dot{\gamma}>c_{2} \Delta \phi^{2}$ [18] are labeled Yield Stress (black) and Critical (blue), consistent with scaling predictions; the Transition regime (red) lies in between. YS data points "peel off" from the master curve (note the black data points above red ones) because it is not possible to collapse three regimes in one plot when their crossovers scale differently.

We stress that the scaling exponents, including dependence on $\Delta \phi$, are all predictions, not adjustable parameters. The excellent data collapse in Figs. $2 \mathrm{~b}$ and 4 is therefore a striking confirmation of the model.

We can gain some intuition for the various regimes by considering different approaches to the critical point (see Fig. 4 inset). Fixing $\phi=\phi_{c}$ and adiabatically lowering the strain rate approaches point $\mathrm{J}$ from the Critical regime $\sigma \sim \gamma_{\text {dyn }}^{2} \sim \dot{\gamma}^{1 / 2}$, where stress is always dominated by dynamic effects. Similarly, fixing $\dot{\gamma}=0^{+}$and adiabatically decreasing $\Delta \phi$ approaches point $\mathrm{J}$ from the Yield Stress regime $\sigma \sim G \gamma_{\mathrm{y}} \sim \Delta \phi^{3 / 2}$, where the flow is rate independent. Finally, there is an anomalous flow regime $\sigma \sim G \gamma_{\text {dyn }} \sim \Delta \phi^{1 / 3} \dot{\gamma}^{1 / 3}$ that transitions between the Critical and Yield Stress regimes. It is traversed when varying $\dot{\gamma}$ at finite $\Delta \phi$ or vice versa.

Length scale - In our model there is no strain rate dependent length scale 4, 10] to capture "swirls" or avalanching rearrangements. To test this assumption, in Fig. 5 a we plot correlations in the non-affine component of the bubble velocities, $C(y):=\left\langle v_{x}(0) v_{x}(y)\right\rangle$. We find $C(y)$ to have a form that is $(i)$ reminiscent of disordered static [19] and quasistatic [7] soft sphere systems and (ii) remarkably robust to changes in $\dot{\gamma}$ and $\phi$. Moreover, as in static linear response [19], $C(y / L)$ collapses for different box sizes $L$, suggesting that for the system sizes studied here the box size is the only relevant macroscopic length scale. We note that $C(y)$ was measured in Ref. [4] in the mean field (MF) bubble model, which replaces realistic bubble-bubble viscous forces by an effective drag term that punishes deviations from an affine (linear) velocity profile - see Fig. 5b. The minimum of $C(y)$ in the MF model (Fig. 5 a inset) selects a length that was found to scale with $\Delta \phi$ and $\dot{\gamma}$ [4. Because this behavior vanishes when the mean field approximation is lifted, we conclude 

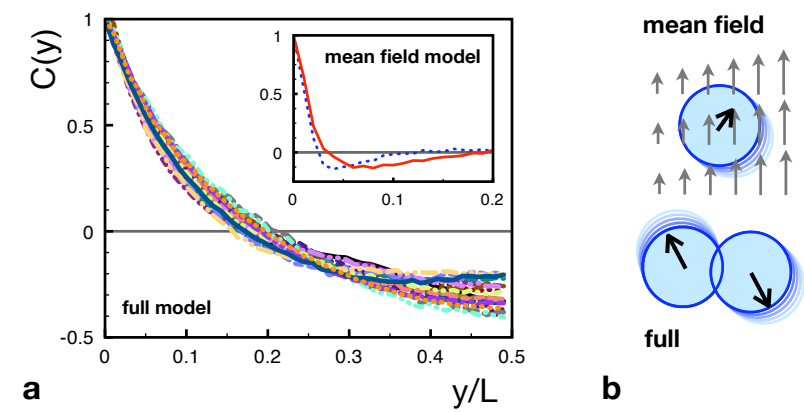

FIG. 5: (a) Two-point correlation function $C(y)$ for $L=75$ (as in Figs. 1 and 4 ), $\dot{\gamma}=10^{-3}$ and $10^{-5}$, and $\phi=0.82$, $\phi \approx \phi_{c}$ and $\phi=0.86$. Also plotted are system sizes $L=54$ and 105 for strain rates $\dot{\gamma}=10^{-3}$ and $10^{-4}$ and packing fractions $\phi=0.82,0.84$, and 0.86. (inset) $C(y)$ has a different form in the mean field model and evolves with $\dot{\gamma}$ and $\Delta \phi$. Here, two examples: $\phi=0.841$ (solid) and 0.88 (dashed) at $\dot{\gamma}=10^{-3}$. (b) Dissipation in the models. Viscous forces (black arrows) are proportional to the difference between a bubble's own velocity and a linear velocity profile (mean field model) or the velocity of a second bubble (full model).

that it is an artifact of the MF dynamics.

Discussion and Outlook - The unanticipated presence of three rate dependent regimes with distinct rationalvalued scaling exponents offers an explanation for dissimilar exponents found in the literature [4, 6, 8, 10, 11, 20]. For data spanning some combination of the Transition, Critical and Viscous regimes, one could fit a power law $\sigma \sim \dot{\gamma}^{\beta}$ with effective exponent $1 / 3<\beta<1$. Reported scalings indeed range from around $\beta \approx 0.4$ [4] to 0.6 [6, 20] and even 1.0 [11. Though the prediction is more difficult to test, the model also provides a plausible argument that the dynamic yield stress of systems with spring-like elastic interactions has super-linear dependence on distance to $\phi_{c}\left(\sigma_{\mathrm{y}} \sim \Delta \phi^{3 / 2}\right)$, rather than linear [8, 21]. Refs. 4, 6] indeed find super-linear scaling, while $\sigma_{\mathrm{y}} \sim \Delta \phi$ in the quasistatic simulations of Ref. [7]. Recent work by Hatano probing the lowest shear rates to date finds $\sigma_{\mathrm{y}} \sim \Delta \phi^{\Delta}$ with $\Delta=1.5 \pm 0.1[23$.

The model is easily generalized to other microscopic interactions. Elastic interactions enter through the scaling of the shear modulus $G \sim \Delta \phi^{\alpha_{\mathrm{el}}-1 / 2}$, while different viscous force laws affect the fluctuations via power balance: $\sigma \dot{\gamma} \sim|\Delta v|^{\alpha_{\text {visc }}+1}$. Recent data for viscous NIPA particles are consistent both with our prediction of $\sigma \sim \dot{\gamma}^{1 / 2}$ in the Critical regime and with a yield stress $\sigma_{\mathrm{y}} \sim \Delta \phi^{\alpha_{\mathrm{el}}+1 / 2}$ for Hertzian interactions, $\alpha_{\mathrm{el}}=3 / 2$ 22]. For physical foams, believed to have a viscous exponent $\alpha_{\text {visc }}=2 / 3$ [24, the Critical regime scales as $\sigma \sim \dot{\gamma}^{2 \alpha_{\text {visc }} /\left(\alpha_{\text {visc }}+3\right)} \sim \dot{\gamma}^{4 / 11}$, in remarkable agreement with recent experiments that found $\sigma \sim \dot{\gamma}^{0.36}$ 24]. Finally, for slow frictional flows, both the the drag forces and the global rheology are rate independent 25. We suggest that the global rate independence is not a triviality and note that it is consistent with our model, where $\sigma \sim \dot{\gamma}^{0}$ for $\alpha_{\text {visc }} \rightarrow 0$.
We thank O. Dauchot, J.M.J. van Leeuwen, A.J. Liu, T.C. Lubensky, M.E. Möbius, S.R. Nagel, P. Olsson, S. Teitel and Z. Zeravcic for helpful interactions. Financial and computational support from the Dutch physics foundation FOM, the Netherlands Organization for Scientific Research, and the National Computing Facilities Foundation are gratefully acknowledged.

[1] A. Liu and S. Nagel, Nature 6706, 21 (1998).

[2] C. O'Hern, L. Silbert, A. Liu, and S. Nagel, Phys. Rev. E 68, 011306 (2003).

[3] M. van Hecke, J. Phys. Cond. Matt. 22, 033101 (2010).

[4] P. Olsson and S. Teitel, Phys. Rev. Lett. 99, 178001 (2007).

[5] T. K. Haxton and A. J. Liu, Phys. Rev. Lett. 99, 195701 (2007); V. Langlois, S. Hutzler, and D. Weaire, Phys. Rev. E 78, 021401 (2008); T. Hatano, Phys. Rev. E 79, 050301 (2009).

[6] T. Hatano, J. Phys. Soc. Japan 77, 123002 (2008).

[7] C. Heussinger and J.-L. Barrat, Phys. Rev. Lett. 102, 218303 (2009).

[8] M. Otsuki and H. Hayakawa, Phys. Rev. E 80, 011308 (2009).

[9] M. Pica Ciamarra and A. Coniglio, Phys. Rev. Lett. 103, 235701 (2009).

[10] A. Lemaître and C. Caroli, Phys. Rev. Lett. 103, 065501 (2009).

[11] D. J. Durian, Phys. Rev. Lett. 75, 4780 (1995); D. J. Durian, Phys. Rev. E 55, 1739 (1997).

[12] In simulations we fix $k=0.35, b=0.025$. Results are presented in dimensionless units where the smaller disk radius $R_{s}$ defines the unit of length, $k$ the unit of stress, and $b / k$ the unit of time.

[13] We determine $\phi_{c}$ and its error bar from the range over which Fig. 4 shows good collapse.

[14] I. K. Ono, S. Tewari, S. A. Langer, and A. J. Liu, Phys. Rev. E 67, 061503 (2003).

[15] All scaling relations refer to typical values.

[16] The square root scaling of $G$ with $\Delta \phi$ (for spring-like interactions) is a hallmark of the static unjamming transition in both isotropic [2, 3, and sheared packings 9.

[17] M. Wyart et al. Phys. Rev. Lett. 101, 215501 (2008).

[18] We select $c_{1}=6$ and $c_{2}=15$.

[19] B. A. DiDonna and T. C. Lubensky, Phys. Rev. E 72, 066619 (2005); C. Maloney, Phys. Rev. Lett. 97, 035503 (2006).

[20] N. Xu and C. S. O'Hern, Phys. Rev. E 73, 061303 (2006).

[21] G. Lois and J. M. Carlson, Europhys. Lett. 80, 58001 (2007).

[22] K. N. Nordstrom et al., Bull. Am. Phys. Soc. 55, X13-9 (2010).

[23] T. Hatano, Prog. Theor. Phys. Suppl. 184, 143 (2010).

[24] G. Katgert, M. E. Möbius, and M. van Hecke, Phys. Rev. Lett. 101, 058301 (2008); G. Katgert et al. Phys. Rev. E 79, 066318 (2009)

[25] P. Schall and M. van Hecke, Ann. Rev. Fluid Mech. 42, 67 (2010). 